\title{
The role of tumor-isolated exosomes on suppression of immune reactions and cancer progression: A systematic review
}

\author{
Mehrdad Pashazadeh*1, 2 (1) \\ Received: 6 Oct 2019 \\ Published: 4 Aug 2020
}

\begin{abstract}
Background: Exosomes are extracellular cells (EVs) emancipated by various cell types and are involved in cell-to-cell transmission. In cancer diseases, exosomes emerge as local and systemic cells to cell mediators of oncogenic information and play a significant role in the advancement of cancer through the horizontal transfer of various molecules, such as proteins and miRNAs.

Methods: In this study, 66 articles from PubMed, MEDLINE, Science Direct, Cochrane, EMBASE, and Scopus were used as English sources.

Results: The biological distribution of cancer cell-derived exosomes in tumor tissue is an important factor in detecting their role in tumor increase; on the other hand, a limited number of studies have examined the biodistribution of exosomes in tumor tissues. While exosomes function as cancer biomarkers and support cancer treatment, we have a long way to improve the antitumor treatment of exosomes and develop exosome-based cancer diagnostic and therapeutic strategies.

Conclusion: This review describes the science and significance of cancer pathogenesis and exosomes relative to cancer treatment resistance.
\end{abstract}

Keywords: Exosome, Cancer pathogenesis, Tumor, Metastasis, Immune system

Conflicts of Interest: None declared

Funding: None

\section{*This work has been published under CC BY-NC-SA 1.0 license. \\ Copyright $\odot$ Iran University of Medical Sciences}

Cite this article as: Pashazadeh M. The role of tumor-isolated exosomes on suppression of immune reactions and cancer progression: A systematic review. Med J Islam Repub Iran. 2020 (4 Aug);34:91. https://doi.org/10.47176/mjiri.34.91

\section{Introduction}

Two different research groups, led by Philip Stahl and Rose Johnstone, independently described reticulocyte fusion back into reticulocyte plasma membranes, and exosomal cargoes were discovered 35 years ago (1). Interestingly, the secretion of exosome mechanism was demonstrated in a study about the iron-binding transferrin receptor by reticulocytes during maturation into erythrocytes in 1983 by Harding et al (1). The downregulation of transferrin receptors was considered as related to endocytosis and lysosomal $\mathrm{d}$ egradation and revealed the multivesicular bodies and fusion with the plasma membrane (2). In recent

Corresponding author:DrMehrdad Pashazadeh, mehrdad_pashazadeh@yahoo.com

1. Immunology Division, Department of Microbiology, Health Science Institute, Bursa Uludag University, Bursa, Turkey

2. Department of Immunology, Faculty of Medicine, Bursa Uludag University, Bursa, Turkey years, a mechanism for intracellular communication involving the extracellular transfer of extracellular vesicles (EVs) has emerged. EVs are composed of both microparticles produced by reverse budding of multi membrane bodies, spilling from the plasma membrane released from nanosicles or exosomes. The microgranules are produced directly from the cell plasma membrane from external cell budding (3). Although the release of apoptotic bodies during apoptosis has been known for a long time (4), the fact that perfectly healthy cells pour vesicles through plasma membranes has only recently been recognized. Most cells,

$\uparrow$ What is "already known" in this topic:

Intercellular communication is essential for all life forms, especially the multicellular organisms. Communication between cells can be achieved by cell-to-cell contact and transmission of specific molecules.

$\rightarrow$ What this article adds:

This review describes the current science and significance in cancer pathogenesis and exosomes relative to cancer treatment resistance. 
including normal and diseased cells, can release the bilayer membrane-bound nanovesicles into the extracellular space and body fluids. EVs derived from this membrane can be divided into 3 main classes depending on their size, following exosomes (40-100 nm), microparticles (100-500 nm), and apoptotic bodies (500-1000 nm). Exosomes are distinguished from apoptotic bodies and microparticles according to their size, origin, and composition. The apoptotic vesicles appear when the cells enter apoptosis as a result of the signals they receive from their environment (5). The protein content of EVs from different sources was analyzed by SDS-PAGE, followed by protein staining, immunoblotting, or proteomic analysis. In highly purified EVs, protein proteins of serum proteins and intracellular compartments (eg, the endoplasmic reticulum or mitochondria) should never contain contaminants that do not come into contact with EVs. As a result, exosomes from different cell types include endosome-related proteins (eg, Lord GTPase, SNAREs, Supplements, and flotillin), some of which are involved in MVE biogenesis (eg, Alix and Tsg101) (6). Membrane proteins, which are known to accumulate in microregions in the plasma membrane or endosomes, are also enriched in EVs. These include tetraspanins, a family of $>$ 30 proteins consisting of 4 transmembrane domains (7-9). The molecular composition of exosomes depends on the cell type of origin, but most exosomes contain highly concentrated molecules. Tetraspanins are the most abundant protein family found on exosomes. Tetraspanin is a highly conserved membrane protein between species, which spans the membrane four times and act as scaffolding proteins, anchoring multiple proteins to a location on the plasma membrane $(8,9)$. The tetraspanins CD9, CD63, CD81, CD82, and CD151 are highly enriched on exosomes in a similar way as lipid rafts (10). Zöller showed that tetraspanins are very important in EVs from other sources (11). Although tetraspanin-enriched membrane regions are different from detergent-resistant lipid, protein rafts, EVs are also enriched in proteins coupled to lipid rafts, including glycosylphosphatidylinositol-associated proteins and flotillin $(12,13)$ (Fig. 1).

In place of, they are formed by inward budding of the endosomal membranes, thereby resulting in the formation of intracellular multivesicular bodies combine and secreted with the plasma membrane (14). Exosomes include blood plasma/serum (15), saliva (16), breast milk (17), cerebrospinal fluid (18), urine, and semen (19). Exosomes are secreted from healthy cells in physiological conditions as well as by cancer cells and tumor-associated stromal cells $(20,21)$. It has been shown that exosomes play an important role in the regulation of immune responses in both innate and adaptive immunity. Exosomes are involved in the spread of self and nonself antigens to many sites of the body due to their small size. Exosomes that are secreted from antigen presenting cells (APCs) such as B cells and dendritic cells (DC) can activate T-cells by direct binding of exosomal MHC-peptide complexes to the T-cell receptor. Also, antigen-bearing exosomes can be internalized and processed by APCs, to activate T-cells (21). Additionally, the origin of the exosomes determines the impact, as mature DC exosomes induce T-cell activation more effectively than immature DC exosomes $(20,21)$. Moreover, exosomes derived from mycoplasma infected DCs could induce both T-cell and B-cell proliferation. Additionally, exosomes derived from mycoplasma infected DCs could induce both Tcell and B-cell proliferation (22). Exosomes are associated with various antigens according to the disease or infection type. Pathogen-infected cell exosomes carry pathogen-specific antigens, which can be processed and associated by DCs to activate T-cells. Furthermore, it has been demonstrated that tumor-derived exosomes can also be processed by DCs and provide antitumoral immune responses by activating T-cells through the presentation of tumor-specific antigens $(22,23)$. The purpose of the article significant in



Fig. 1. Schematic of extracellular vesicles (exosome) (12) 
cancer pathogenesis and exosomes relative to cancer treatment resistance.

\section{Effects of EVs on Suppression of immune responses}

There is great evidence that immune responses are induced by antigen-containing EVs, the release of EVs by tumor cells is a mechanism by which tumors suppress the antitumor immune response. Injection of tumor-derived EVs bearing tumor antigen Ovalbumin (OVA) results in the suppression of delayed-type hypersensitivity (DTH) response to OVA (24). Tumor EVs are likely to be acquired by endogenous APCs that suppress the immune response following the footpad injection of OVA. Similarly, injection of tumor-derived EVs partially induced IL-6 expression in these leader cells by inhibiting the differentiation of $\mathrm{CD}_{1} 1 \mathrm{~b}^{+}$myeloid precursors in the bone marrow $(25,26)$. These myeloid cells have also been shown to alter their pathways of differentiation into a myeloid-derived suppressor cell (MDSC) phenotype (27). Here, some miRNAs found in prostaglandin E2 (PGE2), heat shock protein (HSP70), and tumor-derived EVs play a major role in directing monocyte differentiation towards MDSCs. Also, there is an increase in transforming growth factor beta (TGF- $\beta$ ) expression by myeloid cells that contributes to antigen-specific immunosuppression (27). Interestingly, EVs isolated from the serum of tumor-bearing mice can suppress the immune response to a specific tumor antigen by a mechanism linked to major histocompatibility complex (MHC) class II molecules (MHC-II) (28). Here, the suppression of the antigen-specific immune response is not carried out by tumor-derived EVs, but by $\mathrm{CD} 11 \mathrm{~b}^{+}, \mathrm{MHC}$ class $\mathrm{II}^{+}$host-derived EVs. Thence, in the tumor microenvironment, tumor EVs are assumed to be taken up by macrophages and possibly other APCs and receive EVs that can suppress immune responses to tumor antigens present in tumor EVs. Taken together, these results show that not only tumor-derived EVs can directly suppress $\mathrm{T}$ and natural killer (NK) immune responses, but can also indirectly suppress responses through macrophage cross-presentation and subsequent release of EVs (27).

\section{Exosomes in cancer detection}

Exosomes have been identified in many biological fluids, described in this review above. Cancer exosomes can serve as a fluid biopsy to aid in the diagnosis of malignancies including cancers of the prostate, pancreas, breast, and ovary, glioblastoma, and melanoma (28). Increased exosome concentration has been reported in patients with ovarian, breast, and pancreatic cancer (16). Specific markers associated with cancer exosomes may enhance their enrichment and may be useful for diagnosis when collected from a heterogeneous exosome population in body fluids. In vitro or ex vivo While lipids and metabolites in cancer exosomes provide unique information about cancer detection and biology, more precise information is being developed on the use of proteins and nucleic acids in the exomes. EVs may play an immunosuppressive or immunostimulatory role (7, 8). C-phosphate-G (CPG) has been shown to stimulate B cells from HIV patients to produce lower amounts of $\mathrm{IgG}$ in the presence of EVs from the same patients. Platelet-derived EVs are biased to the secretion of growth factor-secret which causes an anti-inflammatory response and transformation of macrophages. However, exomes containing autoimmune antigens are immunostimulatory in a NOD mouse model and lead to the production of proinflammatory cytokines and the proliferation of $\mathrm{T}$ cells (10). Cancer cell-derived exosomes play a role in determining the tumorigenic potential of epithelial cells $(24,25)$. Cancer ex vivo miR may also contribute to tumor formation and can mediate cell-independent miR biogenesis, which is a feature not associated with normal exosomes. Exosomes with cancer RNA-induced silencing complex-associated miRs induce tumor formation by non-tumorigenic mouse breast cells (MCF-10A cells). In particular, disruption of exosome-associated miR biogenesis by specific silencing of Dicer prevented the growth of MCF10A tumors (25).

\section{Exosomes in tumor angiogenesis}

Exosomes are also involved in the regulation of pathological angiogenesis, including tumor angiogenesis (29). Especially in response to enhanced exosome production due to intratumoral hypoxia, the uptake of cancer cell-derived exosomes by endothelial cells (ECs) stimulates angiogenesis (30). This is achieved in part by stimulating the proangiogenic secretome of ECs (31). Angiogenic programs initiated by hypoxia-induced cell signaling in cancer cells may be affected by exosomes. The effect of tumorderived exosomes on vascular remodeling can affect not only tumor growth but also metastasis. For example, melanoma-derived exosomes cause vascular leakage at sites of metastasis and affect the pro-vasculogenic phenotype of recruited bone marrow (BM) progenitors (32). Cancer cellderived exosomes may also impair the structural integrity of ECs. The uptake of cancer cell-derived exosomal miR105 by ECs lowers the tight fusion protein ZO-1, thereby enhancing vascular permeability and metastatic spread (33).

\section{Effect of exosome in cancer immunotherapy}

Immunotherapy of cancer has recently become one of cancer prevention strategies, particularly for patients who do not respond to conventional treatments (34). These exosomes carried by molecular can be immunostimulatory or immunosuppressive and respectively exosome interactions with the host immune system can lead to a better or worse anti-tumor immune response at the result. Since that is useful for the tumor to suppress anti-tumor immunity, tumor immune microenvironment (TME) will likely support immunosuppression. For this purpose, it is not the only participate in blinded anti-tumor immune responses assembled by the host using the various mechanisms but also interferes with and inhibits anti-tumor immune therapies. Exosomes have recently been shown to exert great effects on immunological activities as mediators involved in many stages of immune and inflammatory responses such as induction, orchestration, subtraction, dissolution, and regulation. As an important example, T regulatory (Treg) Foxp $3^{+}$cells produce exosomes that suppress type $1 \mathrm{~T}$ helper (Th1) cell proliferation and cytokine production by gene silencing due to 
transferred Treg cell-derived miRNAs (27). Also, thymic epithelial cells produce tissue-restricted Antigen-bearing exosomes it directs the improving of Treg cells (28). An illustrative example of allergy relates to $\mathrm{CD} 23^{+}\left(\mathrm{Fc}-\varepsilon \mathrm{R}-2^{+}\right)$ $\mathrm{B}$ cells and then produces exosomes that produce exosomes carrying CD23, IgE, and MHC-class II (B cell exosomes called "bexosomes"), which is transferred to DCs that stimulate antigen-specific $\mathrm{T}$ cells $(28,35)$. Exosome-mediated intercellular interactions involving mast cells are particularly relevant to allergies. These include T-cell exosome activation of mast cells and mast cell activation of $\mathrm{T}$ and $\mathrm{B}$ cells $(36,37)$, exosome-mediated phenotypic and functional maturation of mast cells $(30,38)$, and endothelial cells or progenitor stem cells (39). These effects can be induced by mast cell exhaust transfer of mRNA encoding exosome-mediated cytokines $(36,40)$ or cytokines and by influencing cytokine signaling (40) or by triggering cytokine production by bronchial cells (41) or airway smooth muscle cells (42). Finally, mast cell exosomes induce phenotypic and functional maturation of DC, enabling them to elicit specific immune responses in vivo, activate endothelial cells to secrete endothelial cells clotting factors, and activate $\mathrm{T}$ and $\mathrm{B}$ cells $(36,37)$. Exosomes also participate in various pathological processes such as tumor growth and metastasis, as well as chronic inflammation and infections (43). The list of human diseases mediating pathology is HIV-1 (44), autoimmunity (45), Parkinson's disease (46), Alzheimer's disease (46), numerous inflammatory conditions (47), and others. Perhaps of particular importance to the pathological role of exosomes is their effect on the host immune system. Exosomes produced by malignant or virus-infected cells carry an immunosuppressive cargo and cause dysfunction of the immune effector cells not only locally but also systemically at the site of the disease.

Tumor-derived exosomes suppress immune responses and help advance cancer

During cancer (tumor) development and metastasis, most cancer cells are opposed by the immune system. Suppressing immune reactivation or escaping immune surveillance is a vital task for cancer development. In this process, exosomes produced by both immune and non-immune cells play an important role in regulating host immunity. Most cancer patients have immunosuppression or immunodeficiency. Recently, immunotherapy plays an important role in many cancers such as malignant melanoma, kidney cancer, liver cancer, and even non-small cell lung cancer (48). In this section, we will discuss how exosomes alter the immunoactivity of cancer patients to help cancer cell development. Although tumor exosomes (which) express tumor antigen leading to their recommended use as tumor vaccines, these exosomes also provide antigen-specific immunosuppression (49). A lot of evidence shows the role of exosomes in immunosuppression $(50,51)$. For example, exosomes of OVA expressing melanoma containing full-length OVA protein can effectively suppress an OVA-specific immune response (52). In a tumor-bearing model, plasma-derived exosomes positive for $\mathrm{CD} 11 \mathrm{~b}$ suppressed the tumor antigen-specific response via an MHC class II-linked mechanism (53). The detailed mechanisms of exosomes in suppressing tumor antigen-specific responses have been extensively studied. Tumor-derived exomes not only inhibit the immune activity of multiple lymphoid cell types but also modulate NKG2D expression in cancer to provide immune avoidance $(53,54)$. NKG2D is an activating receptor for $\mathrm{NK}, \mathrm{NKT}$, and $\mathrm{CD}^{+} \mathrm{T}$ cells, and down-regulation of expression or loss in cancer cells is a key mechanism underlying immune avoidance. An important study from Clayton (55) showed that exosomes produced by various cancer cell lines in vitro or isolated from pleural effusions of mesothelioma patients carry NKG2D ligands. These exosomes can trigger the down-regulation of surface NKG2D expression in NK cells and $\mathrm{CD}^{+} \mathrm{T}$ cells. Therefore, lymphocyte activation through NKG2D is impaired after exosome treatment. Furthermore, its interaction with NKG2D ligand positive tumor exosome lymphocytes did not lead to the activation of $\mathrm{CD}^{+} \mathrm{T}$ or NK cells. NKG2D down-modulation was due to $\mathrm{CD}^{+} \mathrm{T}$ of TGF $\beta 1$ or direct exosomes distribution of the NK cell subset (55).

\section{Therapeutic use of exosomes for cancer}

Reports have shown that various clinical studies have demonstrated the role of exosomes as cancer therapies and that several essential side factors have been identified in the application of these nanovesicles in cancer addition. There are effective methods for cancer treatment, for instance, chemotherapy, radiotherapy, hormone therapy, and target therapy. Bone marrow cells, such as breast cancer cells, hair follicle cells, and rapidly dividing cells, are the targets of chemotherapy and radiotherapy (35). Patients with positive estrogen receptor and progesterone receptor have been used hormone therapy (40). The two treatments are limited to side effects on normal cells; therapeutic effects and unpredictable responses. It is also responsible for treatment failure in drug resistance. Exomes with endogenous nanosized membrane vesicles can be used to transport drugs with low immunogenicity and toxicity (31). After modification targeting breast cancer cells, exosomes can be used to give doxorubicin (dox) (33). Exosome derived from epigallocatechin gallate may prevent infiltration of tumor-associated macrophages and polarization of M2 macrophages and thus suppress cancer growth (32). Exosomes have worsened the potential for cancer growth in the tumor microenvironment. Immune escape is an important feature of cancer. Exosomes can transfer anti-tumor substances to ensure the surveillance of the immune system. Exomes released by an adenoviral vector (AdVHER2) showed HER2specific cytotoxic $\mathrm{T}$ lymphocyte responses and dendritic cells (56), which have been shown to confer protective immunity against trastuzumab-resistant breast cancer. It is not appropriate to obtain a large number of exosomes due to the low exosome. Therefore, researchers should look for better methods to produce abundant exosomes without showing large costs. This is also controversial the way exosomes enter the body. Intravenous injection is appropriate, but exosomes should be directed to cancer cells, which is a challenge for exosome design. Avoiding this problem of targeted delivery design with local injection but that is new challenges (57). Exact secretion of exosomes triggers 
changes in the local and systemic tumor environment to induce tumor cells, tumor growth enhancement, metastasis, and insensitivity to drugs. Thus, the destruction of the exosome-propagation pathway through the tumor cells or the removal of these nanovesicles from the blood circulation system can create an effective method of treating cancer (58). Recently, a therapeutic hemofiltration process called ADAPT $^{\mathrm{TM}}$ (adaptive dialysis-like affinity platform technology) has been applied to remove EVs from the bloodstream (59). When the patient's blood plasma samples are transferred via the ADAPT ${ }^{\mathrm{TM}}$ system, the plasma samples factors of the porous fibers are particularly absorbed when interacting with immobilized affinity agents for these target molecules, particularly unbound serum factors and blood cells passing through this system (59). Exosomes are used as vehicles loaded with numerous anticancer drugs, siRNA, and miRNA for many cancer therapeutic burdens. The lipid bilayer membrane of these nanovesicles forms a natural protective shelter, thereby enhancing cellular internalization of encapsulated anti-cancer drugs. For exosomes originating from autologous cancer cells, these nanovesicles may cause minimal toxicity when introduced into target cells and may be less immunogenic than artificial delivery devices. Also, their small size may allow them to escape phagocytosis via the mononuclear phagocyte system (MPS) and can simplify their extravasation through tumor blood vessels and then release in target cancer tissues. Increased colorectal and breast cancers can be suppressed by administering doxorubicin-loaded on exosomes in vivo or using exosome-mimetic nanovesicles. Therefore, the effectiveness of doxorubicin has been extensively supported by targeting immature dendritic cell exosomes to cancer tissues (60). Also, another widely used antimitotic chemotherapeutic drug is Paclitaxel, which can be loaded into microparticles by sonication, and these charged microparticles have 50 times more cytotoxicity than free Paclitaxel for drug-resistant tumor cells in vitro. Besides, the exosomes encapsulated Paclitaxel can substantially block murine Lewis lung cancer, metastases and reduce the size of a tumor in the mouse model (61). Exomes can be used to target specific tissues or organs since they have specific cell tropism according to their properties. The application of a well-characterized exosomal membrane protein (Lamp2b) to express the targeting peptide immediately below the signal peptide sequence containing the targeting peptides rabies viral glycoprotein (RVG) and internalizing RGD (iRGD) was inserted into these nanovesicles to the target brain or cancer tissues from immature dendritic cells (59, $60)$. In addition to anti-cancer therapeutic drugs, exosomes can likewise include several tumor antigens, nano bord, apoptotic-containing proteins, proteasomes, deficient or mutant anti-apoptosis proteins, tumor and tissue-specific peptides, transferrin and lactoferrin in tumor cells for targeting drug (61-65). Exosomes-based cell-free vaccines may indicate alternative dendritic cell therapy to inhibit tumor growth through the function of exosomes in the immune system. These DC-derived exosomes contain costimulatory molecules on MHC-peptide complexes and membranes, that enable them to continue APCs and in- crease immunization in mice compared to antigen-presenting dendritic cells (64). Furthermore, miRNAs are commonly assigned in exosomes in derived cancer cells or isolated from body fluids that contribute to exosome-mediated cell-cell communication and induce anti-cancer properties $(65,66)$.

\section{Conclusion}

Exosomes are an important player in cancer formation and progression due to their biological and morphological features. The facts that they differ according to the cell types they are originated and can be obtained from all fluids in the body are a serious advantage and potential markers for cancer-related studies. Endogenous EVs probably modulate immune responses while stimulating immune responses to antigens, suppressing self-response to antigens. Although EVs can modulate the function of many immune cell types, including $\mathrm{T}$ and $\mathrm{NK}$ cells, the most effective regulatory activity of EVs is achieved by binding or internalizing the cell surface via APCs. In cancer, tumor-derived exosomes carry a large number of immunosuppressive signals, disable anti-tumor immune effector cells, and promote tumor escape from immune control. In cancer, viral infections, autoimmune or other diseases, exosomes that give negative signals to immune cells can interfere with treatment and affect the outcome. However, the fact that exosome biology has not been fully enlightened yet poses a potential obstacle in future studies. In the light of the developing technology, exosomes are thought to be a nano biological structure that deserves to be investigated not only in the formation, progression, diagnosis, and treatment of cancer but also in studies on the mechanism and diagnosis of many diseases.

\section{Conflict of Interests}

The authors declare that they have no competing interests.

\section{References}

1. Harding C, Heuser JE, Stahl P. Exosomes: Looking back three decades and into the future. J Cell Biol. 2013;200(4):367-371.

2. Trams EG, Lauter CJ, Norman Salem J, Heine U. Exfoliation of membrane ecto-enzymes in the form of micro-vesicles. Biochim Biophys Acta. 1981;645(1):63-70.

3. Heijnen H, Schiel A, Fijnheer R, Geuze H, Sixma J. Activated platelets release two types of membrane vesicles: microvesicles by surface shedding and exosomes derived from exocytosis of multivesicular bodies and alpha-granules. Blood. 1999;94(11):3791-3799.

4. Hristov M, Erl W, Linder S. Apoptotic bodies from endothelial cells enhance the number and initiate the differentiation of human endothelial progenitor cells in vitro. Blood. 2004;104:2761-2766.

5.Wickman G, Julian L, Olson M. How apoptotic cells aid in the removal of their own cold dead bodies. Cell Death Differ. 2012;19(5):735-742.

6. Raposo G, Stoorvogel W. Extracellular vesicles: exosomes, microvesicles, and friends. J Cell Biol. 2013;200(4):373-383.

7. Toro J, Herschlik L, Waldner C, Mongini C. Emerging roles of exosomes in normal and pathological conditions: new insights for diagnosis and therapeutic applications. Front Immunol. 2015;6:203.

8. Keller S, Ridinger J, Rupp AK, Janssen JW, Altevogt P. Body fluid derived exosomes as a novel template for clinical diagnostics. J Transl Med. 2011;9:86.

9. Lasser C. Identification and analysis of circulating exosomal microRNA in human body fluids. Methods Mol Biol. 2013;1024:109-128.

10. Raposo G, Stoorvogel W. Extracellular vesicles: Exosomes, microvesicles, and friends. J Cell Biol. 2013:373-383. 
11. Zöller M. Tetraspanins: push and pull in suppressing and promoting metastasis. Nat Rev Cancer. 2009;9:40-55.

12. Thery C, Ostrowski M, Segura E. Membrane vesicles as conveyors of immune responses. Nat Rev Immunol. 2009; 9: 581-93.

13. Wubbolts R, Leckie RS, Veenhuizen PT, Schwarzmann G, Möbius W, Hoernschemeyer J, et el. Proteomic and biochemical analyses of human B cell-derived exosomes. Potential implications for their function and multivesicular body formation. J Biol Chem. 2003;278:1096310972.

14. Sun Y, Liu J. Potential of cancer cell-derived exosomes in clinical application: a review of recent research advances. Clin Ther. 2014;36(6):863-872.

15. Khan S, Jutzy JM, Valenzuela MM, Turay D, Aspe JR, Ashok A, et al. Plasma-derived exosomal survivin, a plausible biomarker for early detection of prostate cancer. PLoS One. 2012;7(10):e46737.

16. Lasser C, Alikhani VS, Ekstrom K, Eldh M, Paredes PT, Bossios A, et al. Human saliva, plasma and breast milk exosomes contain RNA: uptake by macrophages. J Transl Med. 2011;9:9.

17. Admyre C, Johansson SM, Qazi KR, Filen JJ, Lahesmaa R, Norman $\mathrm{M}$, et al. Exosomes with immune modulatory features are present in human breast milk. J Immunol. 2007;179(3):1969-1978.

18. Street JM, Barran PE, Mackay CL, Weidt S, Balmforth C, Walsh TS, et al. Identification and proteomic profiling of exosomes in human cerebrospinal fluid. J Trans1 Med. 2012;10:5.

19. Vojtech L, Woo S, Hughes S, Levy C, Ballweber L, Sauteraud RP, et al. Exosomes in human semen carry a distinctive repertoire of small non-coding RNAs with potential regulatory functions. Nucleic Acids Res. 2014;347.

20. Rabinowits G, Gercel-Taylor C, Day JM, Taylor DD, Kloecker GH. Exosomal microRNA: a diagnostic marker for lung cancer. Clin Lung Cancer. 2009;10(1):42-46.

21. Silva J, Garcia V, Rodriguez M, Compte M, Cisneros E, Veguillas P, et al. Analysis of exosome release and its prognostic value in human colorectal cancer. Genes Chromosomes Cancer. 2012;51(4):409-418.

22. Mincheva-Nilsson L, Baranov V. The role of placental exosomes in reproduction. Am J Reprod Immunol. 2010;63(6):520-533.

23. Vlassov AV, Magdaleno S, Setterquist R, Conrad R. Exosomes: current knowledge of their composition, biological functions, and diagnostic and therapeutic potentials. Biochim Biophys Acta. 2012;1820(7):940-948.

24. Abd Elmageed ZY, Yang Y, Thomas R, Ranjan M, Mondal D, Moroz $\mathrm{K}$, et al. Neoplastic reprogramming of patient-derived adipose stem cells by prostate cancer cell-associated exosomes. Stem Cells. 2014;32(4):983-997.

25. Antonyak MA, Li B, Boroughs LK, Johnson JL, Druso JE, Bryant KL, et al. Cancer cell-derived microvesicles induce transformation by transferring tissue transglutaminase and fibronectin to recipient cells. Proc Natl Acad Sci. 2011;108(12):4852-4857.

26. Melo SA, Sugimoto H, O'Connell JT, Kato N, Villanueva A, Vidal A, et al. Cancer exosomes perform cell-independent microRNA biogenesis and promote tumorigenesis. Cancer Cell. 2014;26(5):707-721.

27. Skogberg G, Lundberg V, Berglund M, Gudmundsdottir J, Telemo E, Lindgren S, Ekwall O. Human thymic epithelial primary cells produce exosomes carrying tissue-restricted antigens. Immunol Cell Biol. 2015;93:727-734.

28. Bretz NP, Ridinger J, Rupp AK, Rimbach K, Keller S, Rupp C, et al. Body fluid exosomes promote secretion of inflammatory cytokines in monocytic cells via TLR signaling. J Biol Chem. 2013;288:3669136702.

29. Hussain HA, Harvey AJ. Evolution of breast cancer therapeutics: breast tumour kinase's role in breast cancer and hope for breast tumour kinase targeted therapy. World J Clin Oncol. 2014;5:299-310.

30. Harding CV, Heuser JE, Stahl PD. Exosomes: looking back three decades and into the future. J Cell Biol. 2013;200:367-371.

31. Tian Y, Li S, Song J. A doxorubicin delivery platform using engineered natural membrane vesicle exosomes for targeted tumor therapy. Biomaterials. 2014;35:2383-2390.

32. Quezada C, Torres Á, Niechi I, Uribe D, Contreras-Duarte S, Toledo $\mathrm{F}$, et al. Role of extracellular vesicles in glioma progression. Mol Aspects Med 2018; 60: 38-51.

33. Dan-dan Y, Ying W, Hong-yu S, Meng-meng L, Wei-xian C, Xiaohui $\mathrm{Z}$, et al. Exosomes in development, metastasis and drug resistance of breast cancer. Cancer Sci. 2015:959-964.

34. Hall J, Prabhakar S, Balaj L, Lai CP, Cerione RA, Breakefield XO. Delivery of therapeutic proteins via extracellular vesicles: review and potential treatments for Parkinson's disease, Glioma, and Schwannoma Cell Mol Neurobiol. 2016;36:417-427.

35. Martin RK, Badrooks KB, Henningsson F, Heyman B, Conrad DH. Antigen transfer from exosomes to dendritic cells as an explanation for the immune enhancement seen by IgE immune complexes. PLoS One. 2014;9:110609.

36. Shefler I, Pasmanik-Chor M, Kidron D, Mekori YA, Hershko AY. T cell derived microvesicles induce mast cell production of IL-24: Relevance to inflammatory skin diseases. J Allergy Clin Immunol. 2014; 133:217-224

37. Shefler I, Salamon P, Reshef T, Mor A, Mekori YA. T Cell-Induced Mast Cell Activation: A Role for Microparticles Released from Activated T Cells. J Immunol. 2010;85:4206-4212.

38. Johnson K, Chu J, Huang M, PowelL M, Villinger F, Bond V. Innate cytokines associate with exosomes in plasma of HIV-1+ individuals (P6205). J Immunol. 2013;190:118.26.

39. Ekström K, Valadi H, Sjöstrand M, Malmhäll C, Bossios A, Eldh M, et al. Characterization of mRNA and microRNA in human mast cellderived exosomes and their transfer to other mast cells and blood CD34 progenitor cells. J Extracell Vesicles. 2012;16:1.

40. Wahlgren J, de Karlson TL, Glader P, Telemo E, Valadi H. Activated Human T Cells Secrete Exosomes That Participate in IL-2 Mediated Immune Response Signaling. PLoS One. 2012;7:49723.

41. Xia YC, Harris T, Stewart AG, Mackay GA. Secreted factors from human mast cells trigger inflammatory cytokine production by human airway smooth muscle cells. Int Arch Allergy Immunol. 2013;160:7585 .

42. Mazzeo C, Canas JA, Zafra MP, Rojas Marco A, Fernandez-Nieto M, Sanz V, et al. Exosome secretion by eosinophils: A possible role in asthma pathogenesis. J Allergy Clin Immunol. 2015;135:1603-1613.

43.Zhang HG, Grizzle WE. Exosomes: a novel pathway of local and distant intercellular communication that facilitates the growth and metastasis of neoplastic lesions. Am J Pathol. 2014; 184:28-41.

44.Teow SY, Nordin AC, Ali SA, Khoo AS. Exosomes in Human Immunodeficiency Virus Type I Pathogenesis: Threat or Opportunity? Adv Virol. 2016;2016:9852494.

45. Tan L, Wu H, Liu Y, Zhao M, Li D, Lu Q. Recent advances of exosomes in immune modulation and autoimmune diseases. Autoimmunity. 2016;49:357-65.

46. Vella L, Hill A, Cheng L. Focus on Extracellular Vesicles: Exosomes and Their Role in Protein Trafficking and Biomarker Potential in Alzheimer's and Parkinson's Disease. Int J Mol Sci. 2016;17:173.

47. Lee H, Zhang D, Zhu Z, Dela Cruz CS, Jin Y. Epithelial cell-derived microvesicles activate macrophages and promote inflammation via microvesicle-containing microRNAs. Sci Rep. 2016;6:35250.

48. Bauernhofer T, Kuss I, Henderson B, Baum AS and Whiteside TL. Preferential apoptosis of CD56dim natural killer cell subset in patients with cancer. Eur J Immunol. 2003;33(1):119-124.

49. Taylor D and Gercel-Taylor C. Tumour-derived exosomes and their role in cancer-associated T-cell signalling defects. $\mathrm{Br} \mathrm{J}$ Cancer. 2005;92(2):305-311

50. Filipazzi P, Bürdek M, Villa A, Rivoltini L, Huber V. Recent advances on the role of tumor exosomes in immunosuppression and disease progression. Seminars in cancer biology: Elsevier. 2012;22(4):342-349.

51. Greening DW, Gopal SK, Xu R, Simpson RJ and Chen W. Exosomes and their roles in immune regulation and cancer. Seminars in cell \& developmental biology: Elsevier. 2015;40:72-81

52. Yang C, Kim SH, Bianco NR and Robbins PD. Tumor-derived exosomes confer antigen-specific immunosuppression in a murine delayedtype hypersensitivity model. PLoS One. 2011;6(8):22517.

53. Yang C, Ruffner MA, Kim SH, Robbins PD. Plasma-derived MHC class II+ exosomes from tumor-bearing mice suppress tumor antigenspecific immune responses. Eur J Immunol. 201242 (7):1778-1784.

54. Viaud S, Terme M, Flament C, Taieb J, Andre F, Novault S, et al. Dendritic cell-derived exosomes promote natural killer cell activation and proliferation: a role for NKG2D ligands and IL-15Ralpha. PLoS One. 2009;4(3):4942

55. Clayton A, Mitchell JP, Court J, Linnane S, Mason MD, Tabi Z. Human tumor-derived exosomes down-modulate NKG2D expression. J Immunol. 2008;180(11):7249-7258.

56. Wang L, Xie Y, Ahmed KA. Exosomal pMHC-I complex targets T cell-based vaccine to directly stimulate CTL responses leading to antitumor immunity in transgenic FVBneuN and HLA-A2/HER2 mice and eradicating trastuzumab-resistant tumor in athymic nude mice. Brest Cancer Res TR. 2013;140:273-284. 
57. Whiteside TL. The tumor microenvironment and its role in promoting tumor growth. Oncogene. 2008;27:5904-12.

58. Gamperl H, Plattfaut C, Freund A, Quecke T, Theophil F, Gieseler F. Ex- tracellular vesicles from malignant effusions induce tumor cell migration: in- hibitory effect of LMWH tinzaparin. Cell Biol Int. 2016;40:1050-1061.

59. Logozzi M, De Milito A, Lugini L, Borghi M, Calabro L, Spada M, et al. High levels of exosomes expressing CD63 and caveolin-1 in plasma of melanoma patients. PloS One. 2009;4:5219.

60. Tian Y, Li S, Song J, Ji T, Zhu M, Anderson GJ, Wei J, et al. A doxorubicin delivery platform using engineered natural membrane vesicle exosomes for targeted tumor therapy. Biomaterials. 2013;35:23832390.

61. Aspe JR, Diaz Osterman CJ, Jutzy JM, Deshields S, Whang S, Wall NR. Enhance-ment of Gemcitabine sensitivity in pancreatic adenocarcinoma by novel exosome- mediated delivery of the Survivin-T34A mutant. J Extracell Vesicles. 2014;3(1):23244.

62. Hung ME, Leonard JN. Stabilization of exosome-targeting peptides via engineered glycosylation. J Biol Chem. 2015;290:8166-8172.

63. Kooijmans SA, Aleza CG, Roffler SR, Van Solinge WW, Vader P, Schiffelers RM. Display of GPI-anchored anti-EGFR nanobodies on extracellular vesicles promotes tumour cell targeting. J Extracell Vesicles. 2016;5(1):31053

64. Malhotra H, Sheokand N, Kumar S, Chauhan AS, Kumar M, Jakhar $\mathrm{P}$, et al. Exosomes: tunable nano vehicles for macromolecular delivery of transferrin and lactoferrin to specific intracellular compartment. J Biomed Nanotechnol. 2016;12:1101-1114.

65. Ohno SI, Takanashi M, Sudo K, Ueda S, Ishikawa A, Matsuyama N, et al. Systemically injected exosomes targeted to EGFR deliver antitumor microRNA to breast cancer cells. Mol Ther. 2013;21:185-191.

66. Yu S, Cao H, Shen B, Feng J. Tumor-derived exosomes in cancer progression and treatment failure. Oncotarget. 2015;6(35):3715137168. 